\title{
Four decades of surgery for bronchogenic carcinoma in one centre
}

\author{
A. Spiliopoulos, M. de Perrot
}

Four decades of surgery for bronchogenic carcinoma in one centre. A. Spiliopoulos, M. de Perrot. (C)ERS Journals Ltd 2000.

ABSTRACT: Since the authors' initial experience in the surgical management of bronchogenic carcinoma in 1956 , more than 40 years have passed. The purpose of this report was to review the authors' data and compare the results by decade (1956-1966; 1967-1976; 1977-1986; and 1987-1996) in order to assess the changing patterns in bronchogenic carcinoma.

A total of 1,597 thoracotomies have been performed. Between the first and last decades of the study, patients' mean age increased from 57 to $63 \mathrm{yrs}$, the ratio of males to females decreased from 19:1 to $3: 1$ and the proportion of adenocarcinoma cases increased from 10 to $34 \%$. The operative mortality decreased from $10 \%$ in 1967- 1976 to $4 \%$ in 1987-1996 and the overall 5-yr survival improved from 27 to $36 \%$ during the same period. The rate of lobectomy progressively increased from 32\% in 1956-1966 to $61 \%$ in 1987-1996, whereas that of pneumonectomy and exploratory thoracotomy decreased from 42 to $28 \%$ and from 20 to $4 \%$, respectively.

Changing patterns of patient characteristics, histology and type of surgery were associated with a constant improvement in the overall 5-yr survival. This improvement was particularly evident among patients with advanced-stage carcinoma. Eur Respir J 2000; 15: 543-546.
Unit of Thoracic surgery, Dept of Surgery, University Hospital of Geneva, Switzerland.

Correspondence: A. Spiliopoulos, Unit of Thoracic Surgery, Dept of Surgery, University Hospital of Geneva, rue Micheli-duCrest 24, 1211 Geneva 14, Switzerland. Fax: 41223727880

\section{Keywords: Lung cancer}

surgery

survival

time trend

Received: April 41999

Accepted after revision December 61999
Since the early 1960s, bronchogenic carcinoma has been the leading cause of cancer-related death among Swiss males [1]. Indeed, the mortality rate has increased from 29 per 100,000 in $1955-1959$ to 65.4 per 100,000 in 1989 1993 [2, 3]. Among Swiss females, the mortality rate increased from 3 per 100,000 in the late 1960 s to 11.6 per 100,000 in 1989-1993 [3, 4]. Lung cancer is now the third commonest cause of cancer-related death among Swiss females after breast and colorectal carcinomas [3].

Although progress has been achieved in adjuvant therapies, surgery still offers the highest probability of a cure in these patients. Since the authors' initial experience in the surgical management of bronchogenic carcinoma in 1956, more than 40 years have passed. The purpose of this report was to review the authors' data since 1956 and to compare the results by decade in order to assess the changing patterns of histology, surgery and outcome in bronchogenic carcinoma.

\section{Material and methods}

The overall survey included all patients who had undergone surgery for bronchogenic carcinoma at the University Hospital of Geneva between November 15, 1956 and December 31, 1996. The University Hospital of Geneva is in a unique position in that since it has always been the only public hospital serving the canton of Geneva, which has $\sim 400,000$ inhabitants. During the four decades in question, the surgical team has comprised two staff surgeons, and all procedures were performed by either one of them or a senior resident under their direct supervision.
Only patients who fulfilled the classification criteria proposed by the World Health Organization [5] were included in the study. All cases of carcinoid tumour, adenoid cystic carcinoma, mucoepidermoid carcinoma and metastatic carcinoma were excluded. Data on age, sex, types of surgical procedure, histology and outcome were retrieved from as far back as 1956.

The preoperative evaluation of tumoral extension evolved considerably during this 40 -yr period. In the authors' initial experience, chest radiography, tomography and bronchoscopy were the only tools available. Thereafter, abdominal ultrasonography and bone scintigraphy were introduced for the detection of distant metastasis, and, since 1984, computed tomographic (CT) scanning of the chest has been used systematically for the further evaluation of local and mediastinal extension of the disease.

Mediastinoscopy has been performed in the authors' institution since 1965. Initially, this procedure was used sporadically, when enlarged mediastinal lymph nodes were suspected on chest radiography or on tomography. With

Table 1. - Characteristics of patients undergoing surgery

\begin{tabular}{lccccc}
\hline & $1956-$ & $1967-$ & $1977-$ & $1987-$ & p-value \\
& 1966 & 1976 & 1986 & 1996 & \\
\hline Patients n & 215 & 253 & 556 & 523 & \\
Sex ratio M:F & $19: 1$ & $14: 1$ & $6: 1$ & $3: 1$ & $<0.0001^{*}$ \\
Age yrs & $57 \pm 9$ & $60 \pm 9$ & $60 \pm 9$ & $63 \pm 10$ & $0.0001^{* *}$ \\
\hline
\end{tabular}

Age date are presented as mean $\pm \mathrm{SD} .{ }^{*}$ : Chi-squared test; **: ttest. M: male; F: female. 


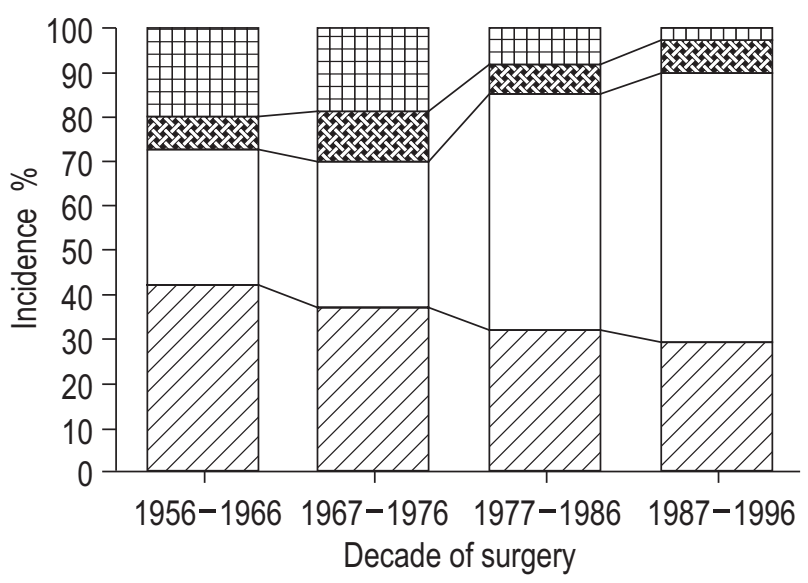

Fig. 1. - Incidence of different types of resection $(\mathbb{Z}$ : pneumonectomy; $\square$ : lobectomy; : segmentectomy; 曲: thoracotomy alone). 1956-1966: $\mathrm{n}=215 ;$ 1967-1976: $\mathrm{n}=253 ; 1977-1986: \mathrm{n}=556 ; 1987-1996: \mathrm{n}=523$. Time trend analysis between 1956-1966 and 1987-1996 was carried out by means of the Chi-squared test for: pneumonectomy, $\mathrm{p}<0.0001$; lobectomy, $\mathrm{p}<0.0001$; segmentectomy, $\mathrm{p}=0.098$; and exploratory thoracotomy, $\mathrm{p}<0.0001$.

the advent of CT scanning, mediastinoscopy has been systematically performed in patients with mediastinal lymph nodes of $>1.5 \mathrm{~cm}$ in their longest diameter.

A staging system was introduced in the late 1960s and, thus, was not available for the first decade of this series. The stages of all patients operated on between 1967 and 1996 were re-evaluated according to the revised "TNM" (primary tumour, regional nodes, metastasis) classification [6].

Radiotherapy was administrated if mediastinal lymph node metastases were observed postoperatively on histological examination or if the resection margins were not microscopically tumour free. Chemotherapy has been added to radiotherapy in patients with mediastinal lymph node metastasis for the last two decades, and, following a randomized controlled trial, in patients with N1 disease since 1995. Induction therapy has been performed since 1997 in patients with N2 disease in the authors' institution; therefore they were not included in this study.

Operative mortality included all deaths occurring during the hospitalization following surgery. Information on follow-up was obtained from hospital charts, telephone calls to general practitioners and review of the regional cancer registry.

\section{Statistical analysis}

Differences between periods were assessed using the Chi-squared test for trend [7]. Continuous data with nor- mal distribution were compared by means of Student's ttest. Survival was analysed using the Kaplan-Meier method [8], and evaluation of the differences was conducted by means of the log-rank test [9]. A p-value of $<0.05$ was regarded as statistically significant.

\section{Results}

Since the first pulmonary resection for bronchogenic carcinoma at the University Hospital of Geneva on November 15, 1956, a total of 1,597 thoracotomies had been performed up to December 31, 1996. These included 503 pneumonectomies, 768 lobectomies, 111 typical or atypical segmentectomies and 165 exploratory thoracotomies. Fifty patients operated on between 1956 and 1976 were excluded from the study because of incomplete data on surgery $(n=6)$, histology $(n=5)$ or both $(n=39)$. The number of patients undergoing surgery increased greatly between the second and third decades of the study and correlated with the creation of an independent section of general thoracic surgery in the authors' institution. The ratio of males to females decreased from 19:1 in the first decade to $3: 1$ in the last decade, and the patients' mean age increased from 57 to 63 yrs during the same period (table 1).

A continuous decrease in the percentage of pneumonectomies was observed, from 42 in the first decade to 28 in the last decade. Concomitantly, the percentage of lobectomies almost doubled from 31 to 61 , whereas lesser resections remained stable at $\sim 7 \%$. Prior to the third decade, the incidence of exploratory thoracotomy was $20 \%$, but declined rapidly thereafter, to $9 \%$ in 1977-1986 and $4 \%$ in 1987-1996 (fig. 1). The reasons for exploratory thoracotomy alone were mainly invasion of mediastinal structures (heart, aorta, superior vena cava or oesophagus) and, occasionally, pleural carcinomatosis not detected preoperatively.

Between 1977 and 1996, mediastinoscopy was performed in 116 patients with bronchogenic carcinoma. Fortyseven of them yielded a negative mediastinoscopy result and underwent surgery. Positive N2 disease was eventually discovered on thoracotomy in nine of these patients. The remaining 69 patients exhibited metastasis-positive mediastinal lymph nodes, homolateral $(n=56)$ or contralateral $(n=13)$ to the tumour. Thoracotomy was performed in eight of these patients with small N2 disease localized in only one level of the mediastinum.

In the authors' initial experience, the operative mortality rates following lobectomy and pneumonectomy were surprisingly low, 7.5 and $6.3 \%$, respectively. In the second decade, the mortality rate increased to $>10 \%$ and declined thereafter during the third and fourth decades. This

Table 2. - Operative mortality rates following surgery

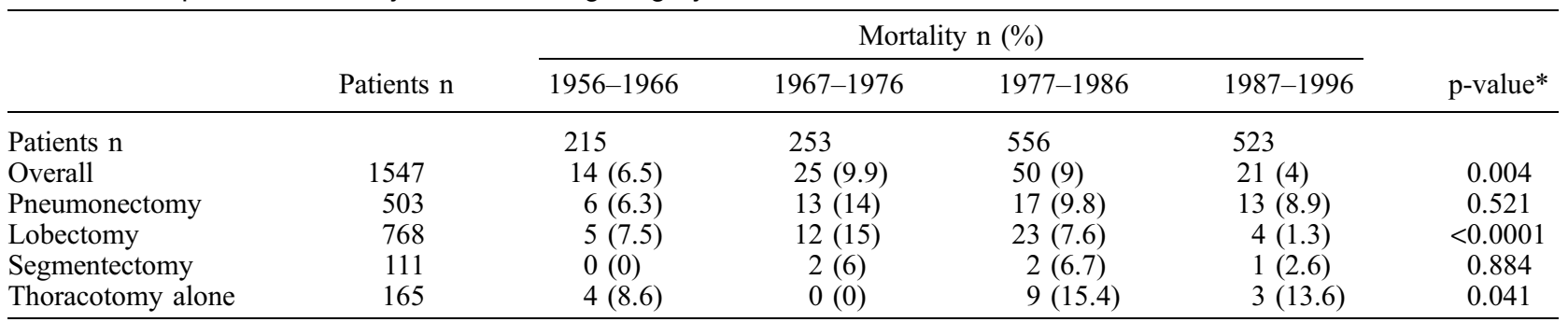

\footnotetext{
*: Time trend analysis by means of the Chi-squared test.
} 


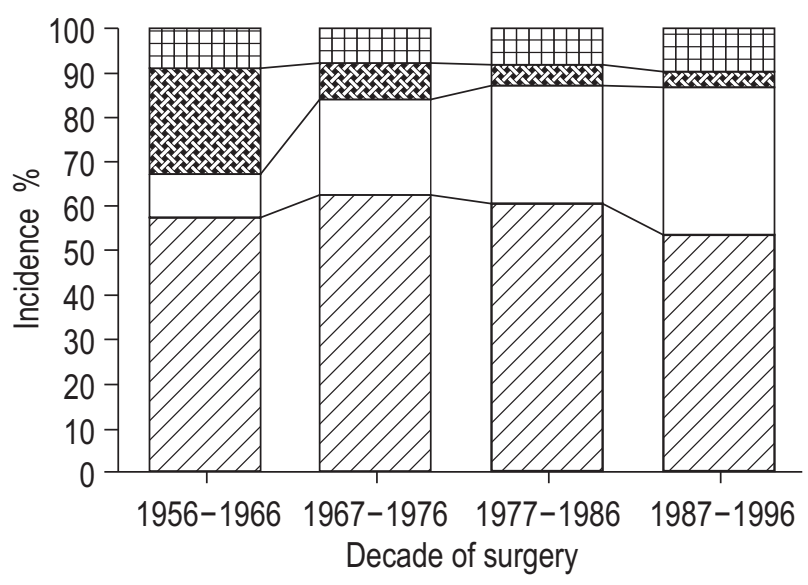

Fig. 2. - Incidence of different types of carcinoma ( $\mathbb{Z}$ : squamous cell; $\square$ : adenocarcinoma; : small cell; 曲: other). 1956-1966: $\mathrm{n}=215$; 1967-1976: $\mathrm{n}=253 ; 1977-1986: \mathrm{n}=556 ; 1987-1996: \mathrm{n}=523$. Time trend analysis between 1956-1966 and 1987-1996 was carried out by means of the Chi-squared test for: squamous cell carcinoma, $\mathrm{p}=0.041$; adenocarcinoma, $\mathrm{p}<0.0001$; small cell carcinoma, $\mathrm{p}<0.0001$; and other carcinomas, $\mathrm{p}=0.633$.

improvement was particularly due to a reduction in the mortality rate following lobectomy, which decreased significantly, from $15 \%$ in $1967-1976$ to $1.3 \%$ in $1987-1996$ (table 2).

Overall, there were 896 squamous cell carcinomas, 403 adenocarcinomas, 103 small cell lung carcinomas and 145 miscellaneous carcinomas. Analysis of the histological trend showed a constant increase in adenocarcinoma from $10 \%$ in the first decade to $34 \%$ in the last decade (fig. 2 ). However, although the incidence of squamous cell carcinoma declined, it was still observed in the majority of patients in the fourth decade. The number of patients undergoing surgery for small cell carcinoma decreased greatly at the end of the 1960 s (from 23 to $8 \%$ ) with the introduction of chemotherapy as the primary treatment modality for this type of tumour.

The overall 5-yr survival progressively increased, from $27 \%$ in $1967-1976$ to $31 \%$ in $1977-1986$ and $36 \%$ in 1987-1996, with a mean follow-up of 38 months for the last decade (fig. 3). This improvement was observed for

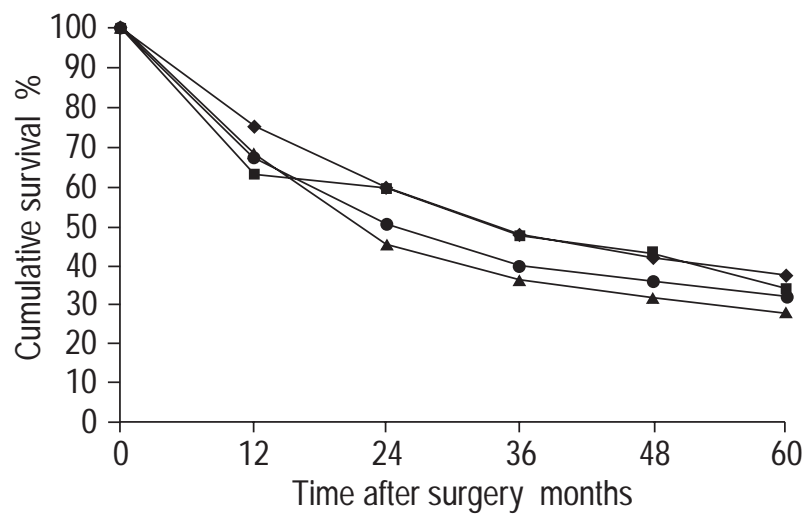

Fig. 3. - Overall 5-yr survival by decade between 1956 and 1996 (exploratory thoracotomies were excluded $\mathbf{\square}$ : 1956-1966 (n=172); $\boldsymbol{\Delta}$ : 1967-1976 (n=204); 0: 1977-1986 ( $=504)$ : 1987-1996 (n=504). Time trend analysis between 1967-1976 and 1987-1996 was carried out by means of the log-rank test: $\mathrm{p}<0.0001$.
Table 3. - Cumulative 5-yr survival according to stage of disease

\begin{tabular}{lcrrrr}
\hline & & \multicolumn{3}{c}{5 -yr survival \% } & \\
\cline { 3 - 5 } Stage & \begin{tabular}{c} 
Patients \\
\cline { 3 - 5 }
\end{tabular} & $1967-$ & $1977-$ & $1987-$ & p-value* \\
& $\mathrm{n}$ & 1976 & 1986 & 1996 & \\
\hline Patients n & & 253 & 556 & 523 & \\
IA & 258 & 59 & 61 & 61 & 0.84 \\
IB & 339 & 39 & 39 & 48 & 0.11 \\
IIA & 61 & 37 & 39 & 47 & 0.51 \\
IIB & 239 & 17 & 18 & 27 & 0.26 \\
IIIA & 265 & 9 & 12 & 19 & 0.01 \\
IIIB & 125 & 0 & 0 & 0 & $* *$ \\
\hline
\end{tabular}

*: Time trend analysis by means of the log-rank test; **: mean survival time increased from 5 months in 1967-1976 to 8 months in $1987-1996(\mathrm{p}=0.01)$.

all stages of carcinoma, but was statistically significant in stage IIIA only (table 3 ). All patients with stage IIIB carcinoma died within 5 yrs after surgery, but the median survival time improved from 5 months in 1967-1976 to 8 months in 1987-1996 ( $\mathrm{p}=0.01)$. The cumulative 5-yr survival significantly improved after lobectomy and segmentectomy, whereas it remained steady after pneumonectomy (table 4).

\section{Discussion}

This series retraces the experience of a single institution in the surgical management of bronchogenic carcinoma over 40 yrs. This period was divided into four decades, which permitted the analysis and comparison of trends in the management and outcome of a large population of patients.

Progressive improvements in the overall cumulative 5yr survival were observed over the last 30 yrs of the study. This contrasts with previous reports from WILKINS et al. [10], who reviewed the first 40 yrs of experience of the Massachussetts General Hospital from 1930 to 1970, and from BELCHER [11], who collected data concerning $>8,000$ patients operated on in England between 1949 and 1980. The discrepancy between these results may be explained by the inclusion of a more recent period in the present analysis. This period certainly benefited from the combined effects of improved treatment modalities and patient selection. Indeed, the effects of a better staging system could explain the improvement in survival observed in all stages of the disease (table 3). However, survival improvement was more pronounced in the group of patients with mediastinal lymph node metastasis, probably because this group of patients also benefited from better adjuvant therapies.

The rate of exploratory thoracotomy significantly declined after the second decade of the present study to reach an incidence of $4 \%$ in the last decade. This finding could be explained by the routine use of CT scanning in the preoperative work-up since the early 1980 s and by the increasing application of mediastinoscopy for patients with suspected mediastinal lymph node metastasis. Concomitantly, the proportion of pneumonectomy cases decreased, presumably because of the increasing incidence of adenocarcinoma, which is usually located more peripherally in the lung, the earlier detection of lung cancer and the increased expertise of the surgical team. 
Table 4. - Cumulative 5-yr survival according to type of disease

\begin{tabular}{|c|c|c|c|c|c|c|}
\hline & \multirow[b]{2}{*}{ Patients $\mathrm{n}$} & \multicolumn{4}{|c|}{ 5-yr survival \% } & \multirow[b]{2}{*}{ p-value* } \\
\hline & & 1956-1966 & $1967-1976$ & $1977-1986$ & 1987-1996 & \\
\hline Patients $n$ & & 215 & 253 & 556 & 523 & \\
\hline Pneumonectomy & 503 & 20 & 24 & 19 & 23 & 0.98 \\
\hline Lobectomy & 768 & 54 & 28 & 38 & 44 & 0.013 \\
\hline Segmentectomy & 111 & 0 & 37 & 27 & 44 & 0.028 \\
\hline Thoracotomy alone & 165 & 0 & 10 & 0 & 6 & - \\
\hline
\end{tabular}

*: Time trend analysis by means of the log-rank test.

The overall operative mortality rate progressively decreased. This improvement certainly resulted from a reduction in the number of pneumonectomies, better selection of patients and improved perioperative care. Pneumonectomies still carry a moderate risk of perioperative death, which it will be difficult to reduce. LiCKER et al. [12] and KOHMAN et al. [13] recently demonstrated that several potential risk factors considered together accounted for only $12-22 \%$ of the mortality rate. Therefore, the death rate of most patients after lung resection remained undetectable preoperatively.

The proportion of females and the incidence of adenocarcinoma increased greatly throughout the study. This increasing incidence of adenocarcinoma was first observed in several regions of the USA in the 1970s [14], and has also recently been reported by European and Asian countries $[1,15]$. This phenomenon is most probably a result of the introduction of filter cigarettes [16]. However, the relation between adenocarcinoma and tobacco use is less strong than that of squamous cell carcinoma or small cell carcinoma, and some authors have postulated that other factors such as viral oncogenes, occupational exposures and dietary factors may have played a role in the new epidemic of lung adenocarcinoma [15].

In conclusion, this series demonstrated that, besides the increasing proportion of females and incidence of adenocarcinoma among thoracotomy patients, the long-term survival of patients undergoing surgery for bronchogenic carcinoma is continuously improving. This improvement was mainly observed among patients with advanced stage carcinoma. Hence, the efficacy of postoperative adjuvant therapy and, more recently, of induction therapy in advanced stage carcinoma should be evaluated in further studies.

\section{References}

1. Levi F, Franceschi S, La Vecchia C, Randimbison L, Te VC. Lung carcinoma trends by histologic type in Vaud and Neuchâtel, Switzerland, 1974-1994. Cancer 1996; 79: 906-914.

2. La Vecchia C, Levi F, Decarli A, Wietlisbach V, Negri E, Gutzwiller F. Trends in smoking and lung cancer mortality in Switzerland. Prev Med 1988; 17: 712-724.
3. Obradovic M, Fioretta G, Droin N, Raymond L, Bouchardy C. Registre genevois des tumeurs. Le cancer à Genève. Incidence, mortalité, survie 1970-1994. Geneva, Département de l'action sociale et de la santé. Registre genevois des tumeurs, 1997.

4. Levi F, Te VC, Randimbison L, La Vecchia C. Trends in cancer incidence and mortality in Vaud, Switzerland, 1974-1993. Ann Oncol 1996; 7: 497-504.

5. World Health Organization. The World Health Organization: histological typing of lung tumors. 2nd Edn. Am J Clin Pathol 1982; 77: 123-136.

6. Mountain CF. Revisions in the international system for staging lung cancer. Chest 1997; 111: 1710-1717.

7. Altman DG. Chi-square for trend. In: Altman DG, ed. Practical Statistics for Medical Research. London, Chapman \& Hill, 1991; pp. 261-265.

8. Kaplan EL, Meier P. Nonparametric estimation from incomplete observations. J Am Statist Assoc 1958; 53: 457481.

9. Peto R, Peto J. Asymptotically efficient rank invariant test procedures. J R Stat Soc (Series A) 1972; 135: 185-198.

10. Wilkins EW, Scannell JG, Craver JG. Four decades of experience with resections for bronchogenic carcinoma at the Massachusetts General Hospital. J Thorac Cardiovasc Surg 1978; 76: 364-368.

11. Belcher JR. Thirty years of surgery for carcinoma of the bronchus. Thorax 1983; 38: 428-432.

12. Licker M, de Perrot M, Höhn L, et al. Perioperative mortality and major cardio-pulmonary complications after lung surgery for non-small cell lung carcinoma. Eur $J$ Cardiothorac Surg 1999; 15: 314-319.

13. Kohman LJ, Meyer JA, Ikins PM, Oates RR. Random versus predictable risks of mortality after thoracotomy for lung cancer. J Thorac Cardiovasc Surg 1986; 91: 551554.

14. Zheng T, Holford T, Boyle P, et al. Time trend and ageperiod-cohort effect on the incidence of histologic types of lung cancer in Connecticut, 1960-1989. Cancer 1994; 74: $1556-1567$.

15. Gao YT, Blot WJ, Zheng W, et al. Lung cancer among Chinese women. Int J Cancer 1987; 40: 604-609.

16. Stellman SD, Muscat JE, Thompson S, Hoffman D, Wynder EL. Risk of squamous cell carcinoma and adenocarcinoma of the lung in relation to lifetime filter cigarette smoking. Cancer 1997; 80: 382-388. 